Helsinki University of Technology Institute of Mathematics Research Reports

\title{
ON A BISECTION ALGORITHM THAT PRODUCES \\ CONFORMING LOCALLY REFINED SIMPLICIAL MESHES
}

Antti Hannukainen Sergey Korotov Michal Křižek 

Helsinki University of Technology Institute of Mathematics Research Reports

\section{ON A BISECTION ALGORITHM THAT PRODUCES \\ CONFORMING LOCALLY REFINED SIMPLICIAL MESHES}

Antti Hannukainen Sergey Korotov Michal Křižek 
Antti Hannukainen, Sergey Korotov, Michal Křižek: On a bisection algorithm that produces conforming locally refined simplicial meshes; Helsinki University of Technology Institute of Mathematics Research Reports A567 (2009).

\begin{abstract}
First we introduce a mesh density function that serves as a criterion to decide, where a simplicial mesh should be fine (dense) and where it should be coarse. Further, we propose a new bisection algorithm that chooses for bisection an edge in a given mesh associated with the maximum value of the mesh density function. Dividing this edge at its midpoint, we correspondingly bisect all simplices sharing this edge. Repeating this process, we construct a family of conforming nested simplicial meshes. We prove that the corresponding mesh size tends to zero for $d=2,3$.
\end{abstract}

AMS subject classifications: 65N50, 65M50

Keywords: bisection algorithm, simplicial elements, conforming finite element method, local refinements, nested meshes

\title{
Correspondence
}

Institute of Mathematics, Helsinki University of Technology

P.O. Box 1100, FI-02015 TKK, Finland

Institute of Mathematics, Academy of Sciences

Žitná 25, CZ-115 67 Prague 1, Czech Republic

antti.hannukainen@hut.fi, sergey.korotov@hut.fi, krizek@math.cas.cz

ISBN 978-951-22-9807-5 (print)

ISBN 978-951-22-9808-2 (PDF)

ISSN 0784-3143 (print)

ISSN 1797-5867 (PDF)

Helsinki University of Technology

Faculty of Information and Natural Sciences

Department of Mathematics and Systems Analysis

P.O. Box 1100, FI-02015 TKK, Finland

email: math@tkk.fi http://math.tkk.fi/ 


\section{Introduction}

Bisection algorithms which refine simplicial partitions were originally used for solving nonlinear equations [5, 17]. Various properties of partitions generated by such algorithms were proved in a number of works in the 70-th [7, 16, 18, 19]. Later, in the 80-th, mainly due to efforts of M. C. Rivara, bisection-type algorithms became popular also in the FEM community for mesh refinement/adaptation purposes $[12,13,14,15]$. Several variants of the algorithm suitable for standard FEMs were also proposed, analysed and numerically tested in $[1,2,3,8,10,11]$ (see also references therein). It has been commonly noticed that inspite of a general simplicity of this type of bisection algorithms, it turns to be hard to provide mesh conformity and simultaneously to prove relevant mesh regularity results $[4,20]$, especially in the case of local mesh refinements and in higher dimensions.

The guiding rules for mesh refinements/adaptivity often come from a posteriori error estimation which generally delivers estimates in the form of integrals over the solution domain. Thus, we usually have in hands certain function over a given domain which dictates the actual mesh reconstruction, see e.g. paper [6]. Its general idea is essentially used in this work, where we propose to apply the longest-edge bisection algorithm, introduced in $[12,13]$ and later analysed in [8], in the following (modified) form. We choose for bisection not the longest edge in the partition, but the edge which has a maximal value of its length multiplied by the value of mesh density function, which is defined a priori. Some properties of such a bisection algorithm are analyzed in this work.

Let $\Omega \subset \mathbf{R}^{d}$ be a bounded polygonal or polyhedral domain. By $\mathcal{T}$ we denote a usual conforming simplicial mesh of $\bar{\Omega}$, i.e., with no hanging nodes. Let $\mathcal{E}=\mathcal{E}(\mathcal{T})$ be the set of all edges of all simplices of $\mathcal{T}$.

A set $\mathcal{F}$ of meshes is said to be a family of meshes if for every $\varepsilon>0$ there exists a mesh $\mathcal{T} \in \mathcal{F}$ such that $|e|<\varepsilon$ for all edges $e \in \mathcal{E}(\mathcal{T})$, where $|\cdot|$ stands for the Euclidean norm.

\section{Mesh density function}

Local simplicial mesh refinements of $\bar{\Omega}$ can be done by means of a priori given positive mesh density function $m$ which is supposed here to be Lipschitz continuous over $\bar{\Omega}$, i.e., there exists a constant $L$ such that

$$
|m(x)-m(y)| \leq L|x-y|, \quad x, y \in \bar{\Omega} .
$$

It is defined to be large over those parts of $\bar{\Omega}$, where we need a very fine mesh. On the other hand, $m$ is defined to be small over those parts of $\bar{\Omega}$, where we require a coarse mesh (see, e.g. Example 2 and Remark 2). From the positiveness and Lipschitz continuity of $m$ we see that there exists a constant $m_{0}$ such that

$$
0<m_{0} \leq m(x) \quad \forall x \in \bar{\Omega} .
$$


Denote by $M_{e}$ the midpoint of any edge $e$ of any mesh of $\bar{\Omega}$ and define the criterion function

$$
J(e)=|e| m\left(M_{e}\right) .
$$

We shall look for an edge $e^{*} \in \mathcal{E}$ (see Remark 1 below) for which $J$ attains its maximal value, i.e.,

$$
J\left(e^{*}\right)=\max _{e \in \mathcal{E}} J(e) .
$$

Further, we find a midpoint $M_{e^{*}}$ of this edge $e^{*}$ and then bisect all simplices from $\mathcal{T}$ sharing $e^{*}$ through the midpoint $M_{e^{*}}$ (see Figs. 1 and 2). For $d=3$ a bisection plane contains the edge opposite to $e^{*}$ in a particular simplex. This refinement strategy will be called the generalized conforming bisection ( $G C B$-) algorithm. It is used repeatedly to produce a family of conforming nested meshes. If $m \equiv 1$ (or if $m$ is constant) then we get the standard conforming longest-edge bisection algorithm which has been recently analyzed in [8], and which refines the mesh globally.

Remark 1 If there exists more than one edge for which $J$ attains its maximum, we may choose $e^{*}$ randomly. (This happens, e.g., if all triangles in $\mathcal{T}$ are equilateral and $m \equiv 1$.) Another way would be to modify slightly the definition of $J$ as follows

$$
J\left(e_{i}\right)=\left|e_{i}\right| m\left(M_{e_{i}}\right)+10^{-10} i, \quad e_{i} \in \mathcal{E}, i=1, \ldots, n,
$$

where $n$ is the number of edges in $\mathcal{E}$.
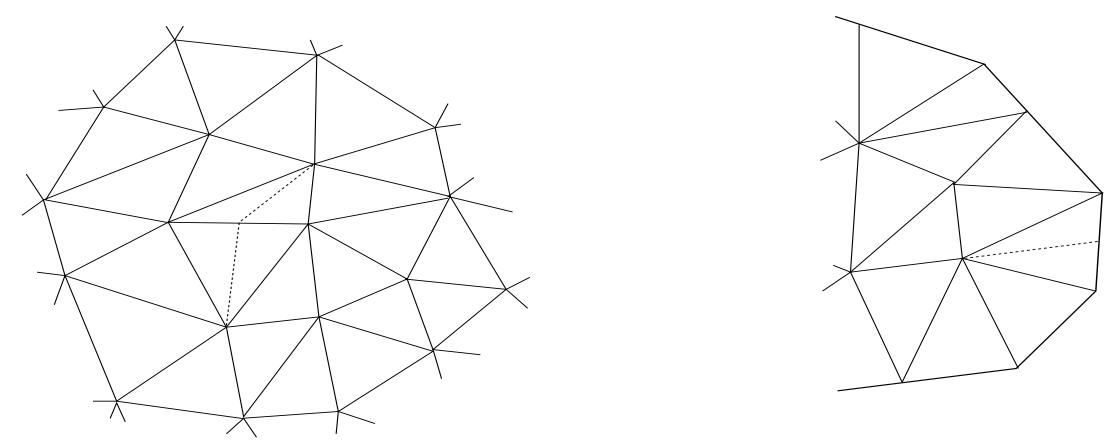

Figure 1: Generalized bisection when $e^{*}$ is inside $\Omega$ and at the boundary $\partial \Omega$ for $d=2$. The dotted lines represent the last bisections.

\section{Convergence of the mesh-size for GCB-algorithm}

In [7], Kearfott proved for the longest-edge bisection algorithm (which however produces hanging nodes, in general, see [8, p. 1688]) that the largest diameter of all simplices tends to zero. In Theorem 2 below we prove the 


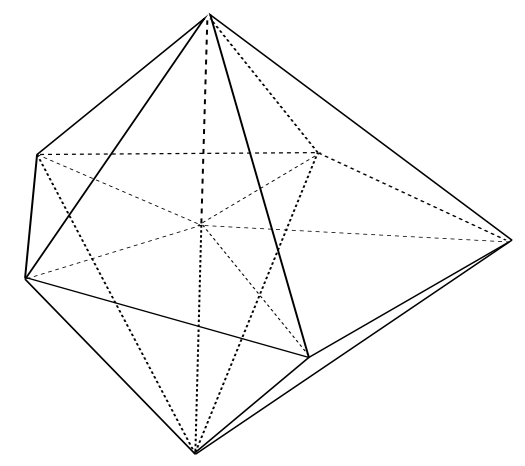

Figure 2: Bisection of all tetrahedra sharing the edge $e^{*}$.

same result for our GCB-algorithm and $d \in\{2,3\}$. Before that we show that the maximal value of $J$ monotonically tends to zero after the "mesh size becomes sufficiently small". The meaning of this expression will be clear from the proof of Theorem 1 (cf. e.g. (7)).

Theorem 1 For each newly generated edge $e^{\prime}$ after one step of the GCBalgorithm applied to $\mathcal{T}$ we always have

$$
J\left(e^{\prime}\right) \leq 0.9 J\left(e^{*}\right)
$$

provided $\left|e^{*}\right|$ is sufficiently small.

P r o o f . Let $e^{*}$ be the edge satisfying (4). Let $T \in \mathcal{T}$ be a triangle which will be bisected for $d=2$. If $d=3$, then $T$ will stand for one of triangular faces containing $e^{*}$ of tetrahedra from $\mathcal{T}$ that will be bisected. There will be three new edges in $T$ : two halves of $e^{*}$ and the median to $e^{*}$. Let $e^{\prime}$ be the first (or second) half of $e^{*}$. Then for a sufficiently small $\left|e^{*}\right|$ we obtain from the positiveness and Lipschitz continuity of $m$ that

$$
0.5 m\left(M_{e^{\prime}}\right) \leq 0.9 m\left(M_{e^{*}}\right) .
$$

We will prove this inequality in detail, since similar inequalities will be used in this proof several times later on. For meshes fine enough we can prove that if

$$
\left|e^{*}\right| \leq \frac{3.2 m_{0}}{L}
$$

then we find by (1) and (2) that

$$
\begin{aligned}
m\left(M_{e^{\prime}}\right) \leq m\left(M_{e^{*}}\right)+L\left|M_{e^{\prime}}-M_{e^{*}}\right|= & m\left(M_{e^{*}}\right)+\frac{1}{4} L\left|e^{*}\right| \\
& \leq m\left(M_{e^{*}}\right)+0.8 m_{0} \leq 1.8 m\left(M_{e^{*}}\right) .
\end{aligned}
$$

Hence, (6) holds provided $\left|e^{*}\right|$ is small (see (7)).

Multiplying (6) by $\left|e^{*}\right|=2\left|e^{\prime}\right|$, we obtain (5), namely

$$
J\left(e^{\prime}\right)=\left|e^{\prime}\right| m\left(M_{e^{\prime}}\right) \leq 0.9\left|e^{*}\right| m\left(M_{e^{*}}\right)=0.9 J\left(e^{*}\right) .
$$


Now let $e^{\prime} \subset T$ be the median to $e^{*}$ and let the lengths of edges $a, b, c$ of $T$ satisfy

$$
|a| \leq|b| \leq|c|
$$

Consider three possible cases:

1) Let $c=e^{*}$ and let $t=e^{\prime}$ be the median on the edge $c$. Since the angle opposite to $c$ is greater than or equal to $\frac{\pi}{3}$, we have

$$
|t| \leq \frac{\sqrt{3}}{2}|c|
$$

Applying (3) and also (9) twice, we find by the Lipschitz continuity of $m$ that

$$
\begin{gathered}
J\left(e^{\prime}\right)=|t| m\left(M_{t}\right) \leq \frac{\sqrt{3}}{2}|c| m\left(M_{t}\right)=\frac{\sqrt{3}}{2}|c| m\left(M_{c}\right)+\frac{\sqrt{3}}{2}|c|\left(m\left(M_{t}\right)-m\left(M_{c}\right)\right) \\
\leq \frac{\sqrt{3}}{2} J(c)+\frac{\sqrt{3}}{2} L|c|\left|M_{t}-M_{c}\right| \leq \frac{\sqrt{3}}{2} J(c)+\frac{\sqrt{3}}{2} L|c| \frac{|t|}{2} \\
\leq \frac{\sqrt{3}}{2} J(c)+\frac{\sqrt{3}}{2} L|c| \frac{1}{2} \frac{\sqrt{3}}{2}|c| \leq \frac{\sqrt{3}}{2} J(c)+\frac{3}{8} L|c|^{2} \leq 0.9 J(c)
\end{gathered}
$$

provided $|c|=\left|e^{*}\right|$ is small enough. Thus, (5) holds.

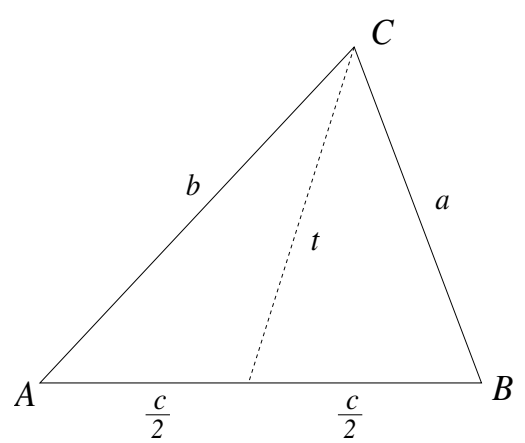

Figure 3: Bisection of a triangle $T \in \mathcal{T}$ for which $c=e^{*}$.

2) Assume now that $b=e^{*}$. Let $u=e^{\prime}$ be the median on $b$. From (8) we can find that (see Fig. 4)

$$
|u| \leq \sqrt{\frac{5}{4}} \frac{1}{\sqrt{2}}|c|=\frac{\sqrt{10}}{4}|c| .
$$

The equality on the left-hand side of (11) is attained when the vertex $B$ is as marked in Fig. 4 and $|a|=|b|$ and $|c|=\sqrt{2}|b|$. Thus,

$$
J(u)=|u| m\left(M_{u}\right) \leq \frac{\sqrt{10}}{4}|c| m\left(M_{u}\right)<\frac{\sqrt{3}}{2}|c| m\left(M_{t}\right),
$$

where the last inequality follows from the positivness and Lipschitz continuity of $m$ like in (6) provided $|b|=\left|e^{*}\right|$ is sufficiently small. From this and (10) we get

$$
J\left(e^{\prime}\right)=J(u) \leq \frac{\sqrt{3}}{2}|c| m\left(M_{t}\right) \leq 0.9|c| m\left(M_{c}\right)=0.9 J(c) \leq 0.9 J(b)=0.9 J\left(e^{*}\right)
$$




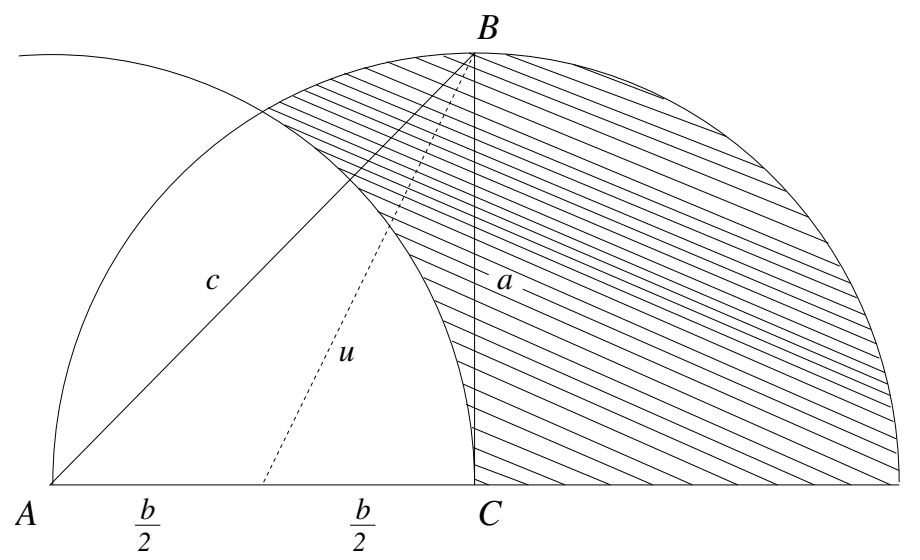

Figure 4: Admissible region for the vertex $B$. The "upper" position of $B$ in this figure yields the maximal value of the ratio $\frac{|u|}{|c|}$.

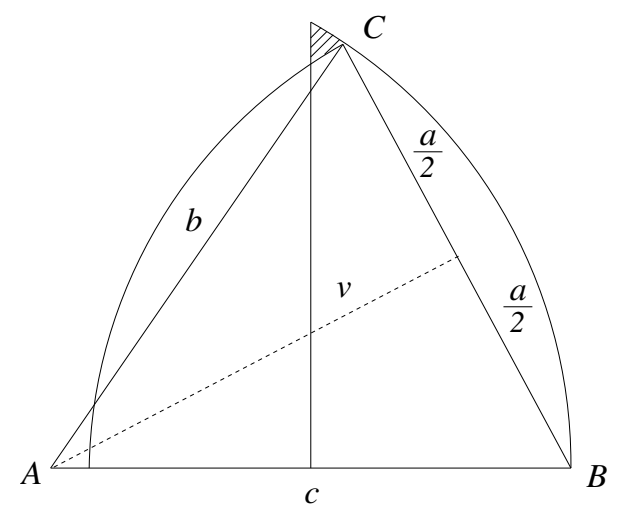

Figure 5: A very small admissible region for the vertex $C$. The position of $C$ in its right corner yields the maximal value of the ratio $\frac{|v|}{|c|}$.

and thus (5) holds again.

3) Finally, let $a=e^{*}$ and let $v=e^{\prime}$ be the median on the edge $a$. Since $J(c) \leq J(a)$, we find by the positivity and Lipschitz continuity of $m$ that

$$
|c| \leq \frac{m\left(M_{a}\right)}{m\left(M_{c}\right)}|a| \leq \frac{10}{9}|a|
$$

if $|a|=\left|e^{*}\right|$ is sufficiently small. From this we get (see Fig. 5)

$$
|v| \leq \sqrt{1-\left(\frac{9}{20}\right)^{2}}|c|=\frac{\sqrt{319}}{20}|c|
$$

The equality on the left-hand side of (12) is attained when the vertex $C$ is in the right corner (where $|a|=\frac{9}{10}|c|$ and $|b|=|c|$ ) of the admissible region marked in Fig. 5.

From (12), the positiveness and Lipschitz continuity of $m$ (cf. (6) again), and (4) we obtain

$$
J\left(e^{\prime}\right)=J(v)=|v| m\left(M_{v}\right) \leq \frac{\sqrt{319}}{20}|c| m\left(M_{v}\right) \leq
$$




$$
\leq 0.9|c| m\left(M_{c}\right)=0.9 J(c) \leq 0.9 J(a)=0.9 J\left(e^{*}\right)
$$

Theorem 2 The GCB-algorithm yields a family of nested conforming meshes whose longest edges tend to zero.

P r o o f . Due to Theorem 1, the value $J\left(e^{*}\right)$ tends monotonically to zero, since for all newly generated edges we have $J\left(e^{\prime}\right) \leq 0.9 J\left(e^{*}\right)$. Therefore,

$$
J\left(e^{*}\right)=\left|e^{*}\right| m\left(M_{e^{*}}\right) \geq|e| m\left(M_{e}\right) \geq|e| m_{0} .
$$

Since $m_{0}$ in $(2)$ is positive, we find that also $|e| \rightarrow 0$.

\section{Numerical results}

Example 1. In [18, 19] Martin Stynes assumes that all triangles are bisected at the same time for $m \equiv 1$. He showed that this repeated process yields only a finite number of similarity-distinct subtriangles, but also hanging nodes may appear. Our algorithm does not produce hanging nodes. Moreover, from Fig. 6 we observe that the number of similarity-distinct subtriangles is also finite if $\Omega$ is a triangle that changes over all possible shapes. This result is proved in $[8, \mathrm{p} .1691]$ under some angle conditions.

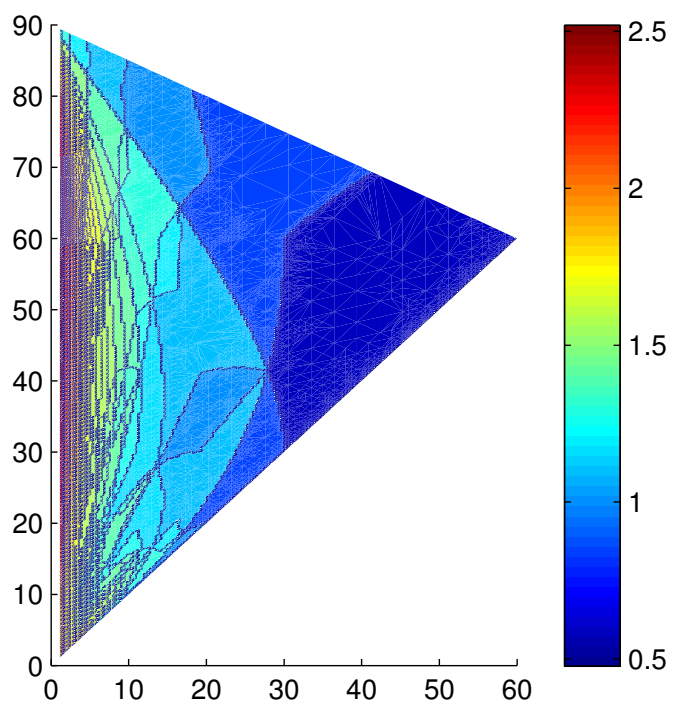

Figure 6: Various colours indicate the value of the decimal logarithm of the number of similarity-distinct subtriangles for $\alpha \leq \beta \leq \gamma, \alpha \in(0, \pi / 3]$, $\beta \in(0, \pi / 2)$ and $m \equiv 1$.

Example 2. Consider the L-shaped domain $\Omega=(-1,1) \times(-1,1) \backslash[0,1) \times$ $[0,1)$. Its initial mesh is illustrated in Fig. 7 . Set

$$
m(x)=\frac{1}{1+4|x|} .
$$


This function and the corresponding meshes from two different iterations are plotted in Fig. 8.

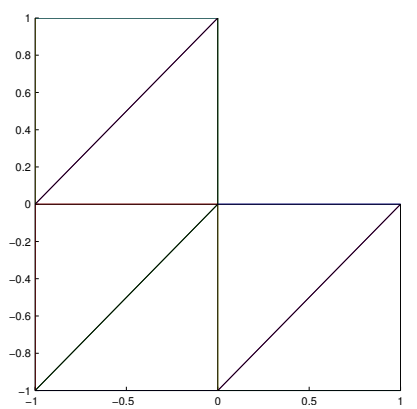

Figure 7: The initial mesh of $\bar{\Omega}$.
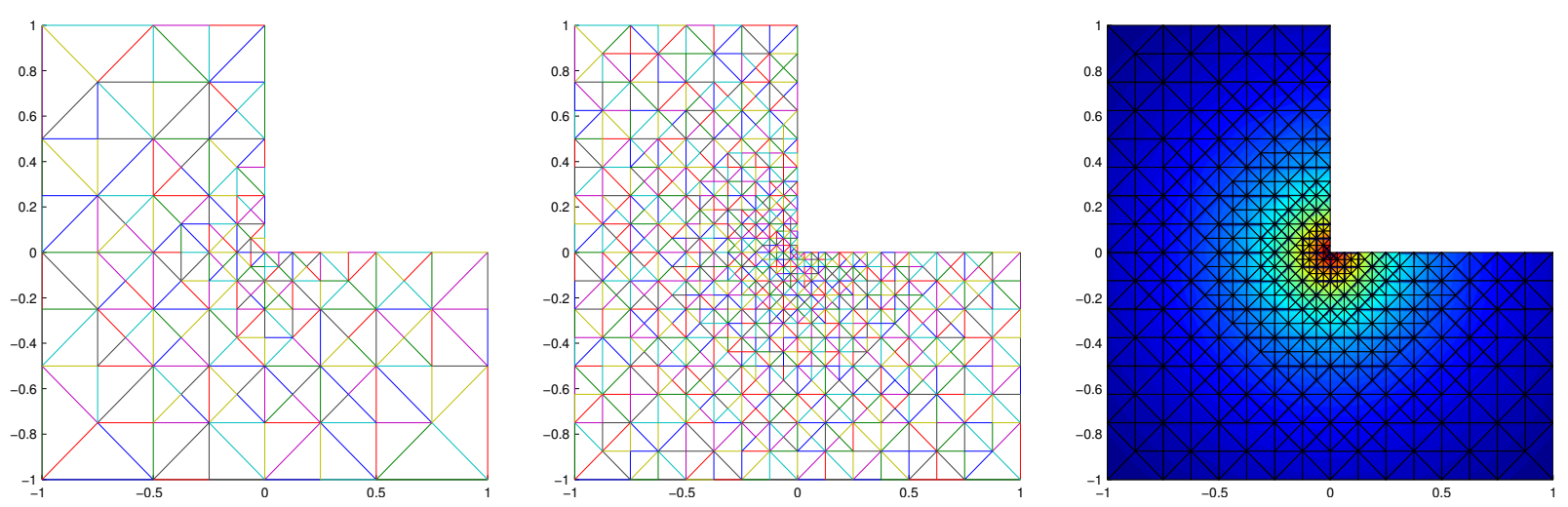

Figure 8: Mesh after 100 and 500 refinements for the mesh density function (13).

Remark 2 In practice, $m$ need not be Lipschitz continuous, but it can have jumps or singularities. The function $m$ can also be modified during computational process. For instance, we may put $m(x)=\left|\tilde{u}(x)-u_{h}(x)\right|$ to control adaptive mesh refinement for a posteriori error estimates. Here $\tilde{u}$ is a higher order approximation of the exact solution of some boundary value problem and $u_{h}$ is a numerical approximation of the true solution. The function $\tilde{u}$ can be obtained, e.g., by some averaging superconvergence technique [9].

Acknowledgement: This paper was supported by Institutional Research Plan nr. AV0Z 10190503 and Grant nr. IAA 100190803 of the Academy of Sciences of the Czech Republic. Also, the support of Projects 211512 and 124619 from the Academy of Finland is acknowledged.

\section{References}

[1] A. Adler. On the bisection method for triangles. Math. Comp. 40 (1983), 571-574. 
[2] D. N. Arnold, A. Mukherjee, and L. Pouly. Locally adapted tetrahedral meshes using bisection. SIAM J. Sci. Comput. 22 (2000), 431-448.

[3] E. Bänsch. Local mesh refinement in 2 and 3 dimensions. IMPACT Comp. Sci. Engrg. 3 (1991), 181-191.

[4] J. Brandts, S. Korotov, and M. Křižek. On the equivalence of regularity criteria for triangular and tetrahedral finite element partitions. Comput. Math. Appl. 55 (2008), 2227-2233.

[5] A. Eiger, K. Sikorski, and F. Stenger. A bisection method for systems of nonlinear equations. ACM Trans. Math. Software 10 (1984), 367-377.

[6] K. Eriksson. An adaptive finite element method with efficient maximum norm error control for elliptic problems. Math. Models Methods Appl. Sci. 4 (1994), 313-329.

[7] R. B. Kearfott. A proof of convergence and an error bound for the method of bisection in $R^{n}$. Math. Comp. 32 (1978), 1147-1153.

[8] S. Korotov, M. Kř́žek, A. Kropáč. Strong regularity of a family of face-to-face partitions generated by the longest-edge bisection algorithm. Comput. Math. Math. Phys. 48 (2008), 1687-1698.

[9] M. Křı̌žek, P. Neittaanmäki. On superconvergence techniques. Acta Appl. Math. 9 (1987), 175-198.

[10] A. Liu and B. Joe. On the shape of tetrahedra from bisection. Math. Comp. 63 (1994), 141-154.

[11] A. Liu and B. Joe. Quality of local refinement of tetrahedral meshes based on bisection. SIAM J. Sci. Comput. 16 (1995), 1269-1291.

[12] M.-C. Rivara. Algorithms for refining triangular grids suitable for adaptive and multigrid techniques. Internat. J. Numer. Methods Engrg. 20 (1984), 745-756.

[13] M.-C. Rivara. Selective refinement/derefinement algorithms for sequences of nested triangulations. Internat. J. Numer. Methods Engrg. 28 (1989), 2889-2906.

[14] M.-C. Rivara and G. Iribarren. The 4-triangles longest-side partition and linear refinement algorithm. Math. Comp. 65 (1996), 1485-1502.

[15] M.-C. Rivara and C. Levin. A 3D refinement algorithm suitable for adaptive and multigrid techniques. Comm. Appl. Numer. Methods Engrg. 8 (1992), 281-290.

[16] I. G. Rosenberg and F. Stenger. A lower bound on the angles of triangles constructed by bisection of the longest side. Math. Comp. 29 (1975), 390-395. 
[17] K. Sikorski. A three dimensional analogue to the method of bisections for solving nonlinear equations. Math. Comp. 33 (1979), 722-738.

[18] M. Stynes. On faster convergence of the bisection method for certain triangles. Math. Comp. 33 (1979), 717-721.

[19] M. Stynes. On faster convergence of the bisection method for all triangles. Math. Comp. 35 (1980), 1195-1201.

[20] M. Zlámal. On the finite element method. Numer. Math. 12 (1968), 394-409. 

(continued from the back cover)

A561 Antti Hannukainen, Sergey Korotov, Michal Krizek

On nodal superconvergence in 3D by averaging piecewise linear, bilinear, and trilinear FE approximations

December 2008

A560 Sampsa Pursiainen

Computational methods in electromagnetic biomedical inverse problems

November 2008

A559 Sergey Korotov, Michal Krizek, Jakub Solc

On a discrete maximum principle for linear $F E$ solutions of elliptic problems with a nondiagonal coefficient matrix

November 2008

A558 José Igor Morlanes, Antti Rasila, Tommi Sottinen

Empirical evidence on arbitrage by changing the stock exchange

December 2008

A556 Lourenço Beirão da Veiga, Jarkko Niiranen, Rolf Stenberg

A posteriori error analysis for the Morley plate element with general boundary conditions

December 2008

A555 Juho Könnö, Rolf Stenberg

Finite element analysis of composite plates with an application to the paper cockling problem

December 2008

A554 Lasse Leskelä

Stochastic relations of random variables and processes

October 2008

A553 Rolf Stenberg

A nonstandard mixed finite element family

September 2008

A552 Janos Karatson, Sergey Korotov

A discrete maximum principle in Hilbert space with applications to nonlinear cooperative elliptic systems

August 2008 
HELSINKI UNIVERSITY OF TECHNOLOGY INSTITUTE OF MATHEMATICS RESEARCH REPORTS

The reports are available at $h t t p: / / m a t h . t k k . f i / r e p o r t s /$.

The list of reports is continued inside the back cover.

A566 Mika Juntunen, Rolf Stenberg

A residual based a posteriori estimator for the reaction-diffusion problem

February 2009

A565 Ehsan Azmoodeh, Yulia Mishura, Esko Valkeila

On hedging European options in geometric fractional Brownian motion market model

February 2009

A564 Antti H. Niemi

Best bilinear shell element: flat, twisted or curved?

February 2009

A563 Dmitri Kuzmin, Sergey Korotov

Goal-oriented a posteriori error estimates for transport problems

February 2009

A562 Antti H. Niemi

A bilinear shell element based on a refined shallow shell model

December 2008

ISBN 978-951-22-9807-5 (print)

ISBN 978-951-22-9808-2 (PDF)

ISSN 0784-3143 (print)

ISSN 1797-5867 (PDF) 